\title{
The origin and evolution of plant cystatins and their target cysteine proteinases indicate a complex functional relationship Manuel Martinez* and Isabel Diaz
}

\author{
Address: Laboratorio de Bioquímica y Biología Molecular, Dpto. de Biotecnología-Centro de Biotecnología y Genómica de Plantas-Universidad \\ Politécnica de Madrid, ETS Ingenieros Agrónomos, Ciudad Universitaria s/n. 28040 Madrid, Spain \\ Email: Manuel Martinez* - m.martinez@upm.es; Isabel Diaz - i.diaz@upm.es \\ * Corresponding author
}

Published: 10 July 2008

BMC Evolutionary Biology 2008, 8:198 doi:10.1/86/|47|-2/48-8-198

This article is available from: http://www.biomedcentral.com//47/-2/48/8/I98

(c) 2008 Martinez and Diaz; licensee BioMed Central Ltd.

This is an Open Access article distributed under the terms of the Creative Commons Attribution License (http://creativecommons.org/licenses/by/2.0), which permits unrestricted use, distribution, and reproduction in any medium, provided the original work is properly cited.
Received: 12 February 2008

Accepted: 10 July 2008

\begin{abstract}
Background: Cystatins and their putative targets, the families of cysteine proteinases $\mathrm{CIA}$ and $\mathrm{Cl} 3$ play key roles in plants. Comparative genomic analyses are powerful tools to obtain valuable insights into the conservation and evolution of the proteinases and their proteinaceous inhibitors, and could aid to elucidate issues concerning the function of these proteins.

Results: We have performed an evolutionary comparative analysis of cysteine proteinases CIA and $\mathrm{Cl} 3$ and their putative inhibitors in representative species of different taxonomic groups that appeared during the evolution of the Viridiplantae. The results indicate that whereas CIA cysteine proteinases are present in all taxonomic groups, cystatins and $\mathrm{Cl} 3$ cysteine proteinases are absent in some basal groups. Moreover, gene duplication events have been associated to the increasing structural and functional complexities acquired in land plants.

Conclusion: Comparative genomic analyses have provided us valuable insights into the conservation and evolution of the cystatin inhibitory family and their putative targets, the cysteine proteinases from families $\mathrm{CIA}$ and $\mathrm{CI}$. Functionality of both families of proteins in plants must be the result of a coevolutionary process that might have occurred during the evolution of basal and land plants leading to a complex functional relationship among them.
\end{abstract}

\section{Background}

Proteinaceous peptidase inhibitors are proteins that have the potential to attenuate the activities of peptidases by the formation of complexes with the enzymes. In the MEROPS database (release 8.00), 56 different families of peptidase inhibitors are included $[1,2]$. One of them corresponds to a family of peptidase inhibitors called cystatins, which constitute a superfamily of evolutionary related proteins able to inhibit cysteine proteinases from the papain subfamily C1A. Those from plants are called phytocystatins (PhyCys) and form an independent subfamily that cluster on a distinct branch from other cystatin fami- lies on the phylogenetic tree [3]. The cystatin inhibitory mechanism involves a wedge formed by the partially flexible N-terminus containing a glycine residue and two hairpin loops carrying a highly conserved motif QXVXG and a tryptophan residue, respectively $[4,5]$. Most PhyCys have a molecular mass in the $12-16 \mathrm{kDa}$ range and are devoid both, of disulphide bonds and of putative glycosilation sites. However, several PhyCys with a molecular mass of $\approx$ $23 \mathrm{kDa}$ have a carboxy-terminal extension, which has been involved in the inhibition of a second family of cysteine peptidases, the legumain peptidases C13 [6]. PhyCys have a dual role. In the plant, they have been 
related to the regulation of activity of endogenous cysteine proteinases during seed development and germination [7-10], and of programmed cell death [11,12]. Furthermore, a defense role has been inferred to PhyCys from their ability to inhibit exogenous proteinases such as those present in the digestive tracts of insects [13-15], the enhanced resistance against pests observed in transgenic plants overexpressing PhyCys genes [15-18], and the antifungal activities described for certain PhyCys [19-23].

The main target of PhyCys, the papain-like subfamily C1A is the most thoroughly studied among plant cysteine proteinases. Papain contains three disulfide bonds and its chain is folded to form a globular protein with two interacting domains delimiting a cleft at the surface where substrates can be bound [24]. The evolutionary highly conserved catalytic mechanism of these peptidases involves the three amino acids Cys 25, His 159 and Asn 175 (according to the papain numbering). These enzymes are synthesized as inactive precursors, which comprise an $\mathrm{N}$-terminal signal peptide, a 38-250 residues prosequence, and the mature protein generally 220-260 amino acids long. Activation takes place by limited intra- or intermolecular proteolysis cleaving off an inhibitory propeptide [25]. In plants, papain-like peptidases are involved in various physiological processes, such as the post-translational processing of storage proteins into mature forms and the liberation of amino acids to be used during germination [26-29]. An important role in the intracellular catabolism for senescence and programmed cell death has been also attributed to papain-like enzymes [11,30,31]. Moreover, a role in stress tolerance and defence against pathogens has been postulated [32-34].

The second target to cystatins is the legumain-like family C13 of cysteine proteinases. Their tertiary structure has not been reported yet, but similarities in sequence and predicted secondary structure around their active site residues Histidine and Cysteine lead Chen et al. [35] to suggest that their fold is similar to that of the caspases in family C14. Legumain is synthesized as a precursor with both $\mathrm{N}$ - and C-terminal propeptides. Prolegumain is directed to the plant vacuole, where activation occurs at least partially by autolysis [36]. In plants, legumains or VPE (vacuolar processing enzymes) catalyze an Asn- and Asp-specific limited proteolysis. There is abundant evidence indicating that legumains perform a proteinprocessing function that causes a limited proteolysis of precursor proteins [37]. Legumain from plant seeds is thought to be responsible for the post-translational processing of seed proteins prior to storage [38]. During germination, legumains contribute to the activation of cysteine proteinases to degrade storage proteins $[39,40]$. A role in defense against pathogens executing programmed cell death due to the caspase activity observed for several legumains has also been proposed [41,42].

Although peptidase-inhibitor interactions are crucial to many important processes in plant cells, very little information is available on the origin and evolution of these protein families in plants. To date, a comprehensive analysis of cystatin families has been only done in the completed genomes of the mono and dicotyledoneous model plants Oryza sativa and Arabidopsis thaliana [43]. Likewise, the family of papain-like cysteine proteinases from Arabidopsis was formerly described by Beers et al. [44], and different families of putative cysteine proteinases in the completely sequenced genomes of Populus trichocarpa and Arabidopsis have been reviewed by Garcia-Lorenzo et al. [45].

Comparative genomic analyses could provide valuable insights into the conservation and evolution of the proteinases and their proteinaceous inhibitors, which could aid to elucidate issues concerning the function of these proteins. Thus, we have performed a phylogenetic analysis of these gene families in representative species of different taxonomic groups. The results indicate that whereas papain-like cysteine proteinases are present in all taxonomic groups, cystatins and legumain-like proteins are absent in some basal groups. Moreover, gene duplication events have been associated to the increasing structural and functional complexities acquired in land plants.

\section{Results \\ A survey of cystatins and cysteine proteinases (CIA and CI3) in plants}

In a first step to know the origin and evolution of cystatins and papain and legumain-like cysteine proteinases in plants, an extensive search of the TIGR plant transcript assemblies (TA) database was made. All plant species for which more than 1,000 ESTs or CDNA sequences are available are included in the database. Thus, TAs from 252 plant species from unicellular organisms to flowering plants could be explored. In addition, the Tree of Life Web Project provides information on the evolutionary phylogenetic relationships in the green plants. Based on this information, we constructed a tree with the main clades of modern plants (Fig. 1). In this tree, the presence of any species of each clade with a TA belonging to the cystatin family, or to the papain-like or legumain-like cysteine proteinase families is represented. The clades of the Embryophytes or land plants present cystatins and both types of cysteine proteinases, with the exception of Cycads and Gingkos, in which no legumain-like cysteine proteinases have been detected yet. In contrast, the algae have a great variability of detected proteinases and inhibitors. Thus, whereas cystatins have not been detected in Prasinophytes, Ulvophyceae and Zignematales, the algae groups 


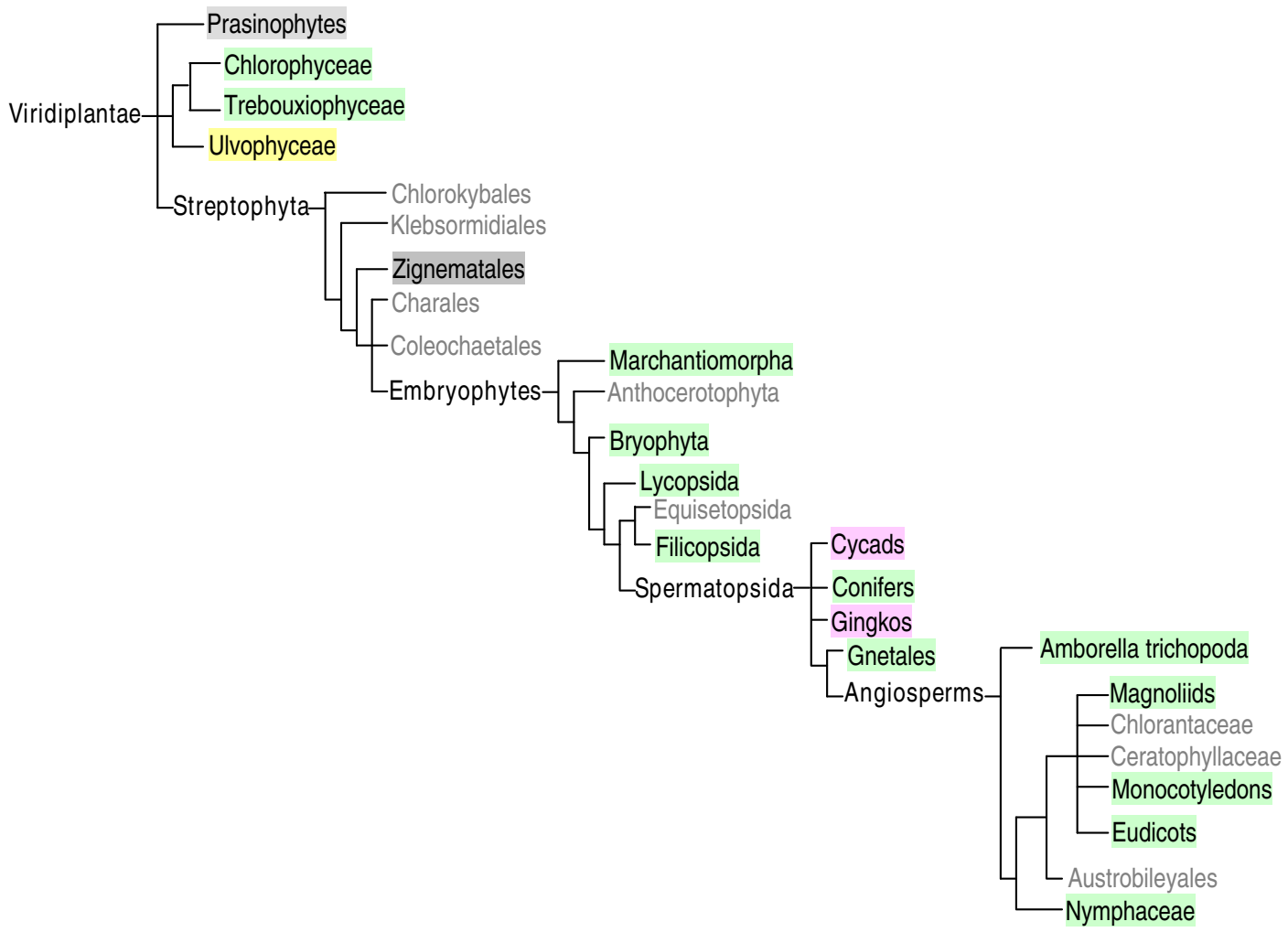

\section{Figure I}

Schematic evolutionary tree of Viridiplantae based on the Tree of Life Web Project. Different colours indicate the presence of any modern species in the clade with at least one transcript assembly belonging to the cystatin family, the papainlike CIA, and/or the legumain-like CI 3 cysteine proteinase families. Green, presence of cystatin, CIA and CI3 proteins. Grey, presence of $\mathrm{CIA}$ and $\mathrm{CI} 3$ proteins. Yellow, presence of CIA proteins. Pink, presence of cystatin and CIA proteins. Absence of colour indicates clades without representative species in the TIGR plant transcript assemblies database.

Chlorophyceae and Trebouxiophyceae possess both proteinases and inhibitors. This approximation permitted us a survey of how these protein families could have evolved. However, the actual number of papain and legumain-like proteinases and cystatins encoded in a plant genome can only be known using the whole genome sequence.

\section{Number of cystatins and cysteine proteinases (CIA and CI3) in completely sequenced plants}

To date, four algae (two Prasinophytes, Ostreococcus tauri and Ostreococcus lucimarinus; and two Chlorophyceae, Chlamydomonas reinhardtii and Volvox carteri), one moss (Physcomitrella patens), one spikemoss (Selaginella mollendorffii), and three angiosperms (Arabidopsis thaliana, Populus trichocarpa and Oryza sativa) have been completely sequenced and drafts of these sequences are available on the web. These eight species and the monocot species Hordeum vulgare, with an extremely large number of EST and cDNA sequences available, were selected to establish the number of proteinases and inhibitors. Thus, extensive searches for C1A, C13 proteinases, and cystatins were made (Table 1). Among the algae, no cystatins were found in the two Ostreococcus species, whereas the Chlorophyceae species possess a unique cystatin that lacks the $3^{\prime}$ extension related to the inhibitory properties against legumain-like proteinases. However, all these species present papain-like proteinases and the Chlorophyceae species a unique legumain proteinase. From the moss to the angiosperms, the number of cystatins gradually increased. In the moss and the spikemoss, the presence of cystatins with the 3 ' extension is correlated with an increase in the number of legumain-like proteins. In angiosperms, a higher number of cystatins than in the non-seed land plants is accompanied by a sharp increase in the number of papain-like cysteine proteinases.

\section{Evolution of CIA cysteine-proteinases in plants}

To know how papain-like cysteine proteinases could have evolved from algae to angiosperms, the C1A mature protein fragments from 25 amino acids before the putative location of the cysteine reactive residue until 18 amino acids after the asparagine reactive residue were selected. 
Table I: Number of CIA and CI3 cysteine proteinases and their putative inhibitors.

\begin{tabular}{lccc}
\hline Organism & Cystatins (with 3' extension) & Papain-like proteinases & Legumain-like proteinases \\
\hline Ostreococcus tauri & $0(0)$ & 9 & 0 \\
Ostreococcus lucimarinus & $0(0)$ & 9 & 0 \\
Chlamydomonas reinhartdii & $1(0)$ & 11 & 1 \\
Volvox carteri & $1(0)$ & 14 & 1 \\
Physcomitrella patens & $5(3)$ & 11 & 4 \\
Selaginella moellendorffii & $2(1)$ & 45 & 2 \\
Oryza sativa & $12(1)$ & 32 & 5 \\
Arabidopsis thaliana & $7(2)$ & 36 & 4 \\
Populus trichocarpa & $8(3)$ & 32 & 5 \\
Hordeum vulgare & $13(1)$ & 5 \\
\hline
\end{tabular}

These amino acid sequences were aligned by MUSCLE (see Additional file 1, partial sequences corresponding to HvPap-23 and HvPap26 were not included), and a phylogenetic tree was constructed by the maximum likelihood PhyML method (see Additional file 2). A schematic representation of the obtained cladogram is shown in Figure 2. C1A proteinases were originally described as cathepsins in mammals, and plant cysteine proteinases have been traditionally classified based on their homology to them [44]. When the obtained $\mathrm{C} 1 \mathrm{~A}$ proteins were classified in this form, ten main groups comprising $\mathrm{C} 1 \mathrm{~A}$ proteinases homologous to the human $\mathrm{B}, \mathrm{F}, \mathrm{H}$, and L-cathepsins were found. B-like proteins shared a similar sequence to human B-cathepsin. F-like proteinases conserved an ERFNAQ motif typical to this kind of cathepsins in their proregion. $\mathrm{H}$ and L-like proteins conserved the ERFNIN motif present in mammal $\mathrm{H}$ and $\mathrm{L}$ cathepsins in the proregion. Several algal papain-like proteinases (groups Algae A to D) and the barley protein HvPap-32 did not present enough similarities to mammalian cathepsins to be considered as their plant homologous proteins and were grouped separately. To deal with the extremely complex group of $\mathrm{H}$ and L-like cathepsins, an analysis to discover homologous motifs was made using the MEME software (data not shown). All the proteins shared a high number of identical motifs, but several ones could be used to discriminate among proteins belonging to different subgroups. From this analysis, five subgroups (A to E) of Llike proteins were defined. Two of them (B and C) correlated with well-defined branches of the phylogenetic tree and were formed by only angiosperm proteins, as well as group A which was split in several branches. Group D includes branches of L-like C1A sequences composed of algae, moss, spikemoss and angiosperm proteins. Three branches of the phylogenetic tree formed by cereal sequences could be assigned to the subgroup E. Some other branches formed by $\mathrm{C} 1 \mathrm{~A}$ proteins for all clades could not be included to any subgroup and were assigned as other L-like cathepsins (Suppl. Fig. 1). For H-like cathepsins, two subgroups could be detected, one of them included C1A proteinases from the moss to angiosperms, and the other was formed by algal proteins. In Table 2, the number of $\mathrm{C} 1 \mathrm{~A}$ proteinases belonging to each group is shown. B-like C1A are present in all analyzed species and the number did not vary during plant evolution. F-like proteinases were also maintained from algae, although they were not detected in the Chlorophyceae, and their number slightly increased in angiosperms. H-like proteinases were detected in Chlorophyceae, and were maintained from moss to angiosperms. L-like proteins showed the most striking pattern. They first appeared in algae, slightly increased in the moss, and they presented a sharp increase from the spikemoss to the angiosperms. Finally, C1A proteinases not related to mammalian proteinases (indicated as other) were mainly found in algae.

\section{Evolution of $\mathrm{CI} 3$ cysteine-proteinases in plants}

Similarly, to know how the second target of cystatins, the legumain-like cysteine proteinases could have evolved from algae to angiosperms, the amino acid sequences of C13 proteinases were aligned by MUSCLE (see Additional file 1), and a cladogram was constructed by the maximum likelihood PhyML method (Fig. 3). The C13 proteins from Chlorophyceae were the most different and did not grouped with the other legumain sequences. Orthology relationships could be detected for angiosperm proteins located in groups supported by approximate likelihood-

Table 2: Number of CIA cysteine proteinases grouped by similarity to mammalian proteinases.

\begin{tabular}{lccccc}
\hline Organism & B-like & F-like & H-like & L-like & Others \\
\hline Ostreococcus tauri & 3 & $\mathrm{I}$ & 0 & 2 & 3 \\
Ostreococcus lucimarinus & 3 & $\mathrm{I}$ & 0 & 2 & 3 \\
Chlamydomonas reinhartdii & 3 & 0 & 2 & 2 & 4 \\
Volvox carteri & 2 & 0 & 2 & 2 & 8 \\
Physcomitrella patens & 3 & 2 & $\mathrm{I}$ & 5 & \\
Selaginella moellendorffii & $\mathrm{I}$ & 3 & $\mathrm{I}$ & 14 & \\
Oryza sativa & $\mathrm{I}$ & 3 & $\mathrm{I}$ & 40 & \\
Arabidopsis thaliana & 3 & 3 & 2 & 24 & \\
Populus trichocarpa & 3 & 5 & $\mathrm{I}$ & 27 & \\
Hordeum vulgare & 2 & 3 & $\mathrm{I}$ & 25 & $\mathrm{I}$ \\
\hline
\end{tabular}




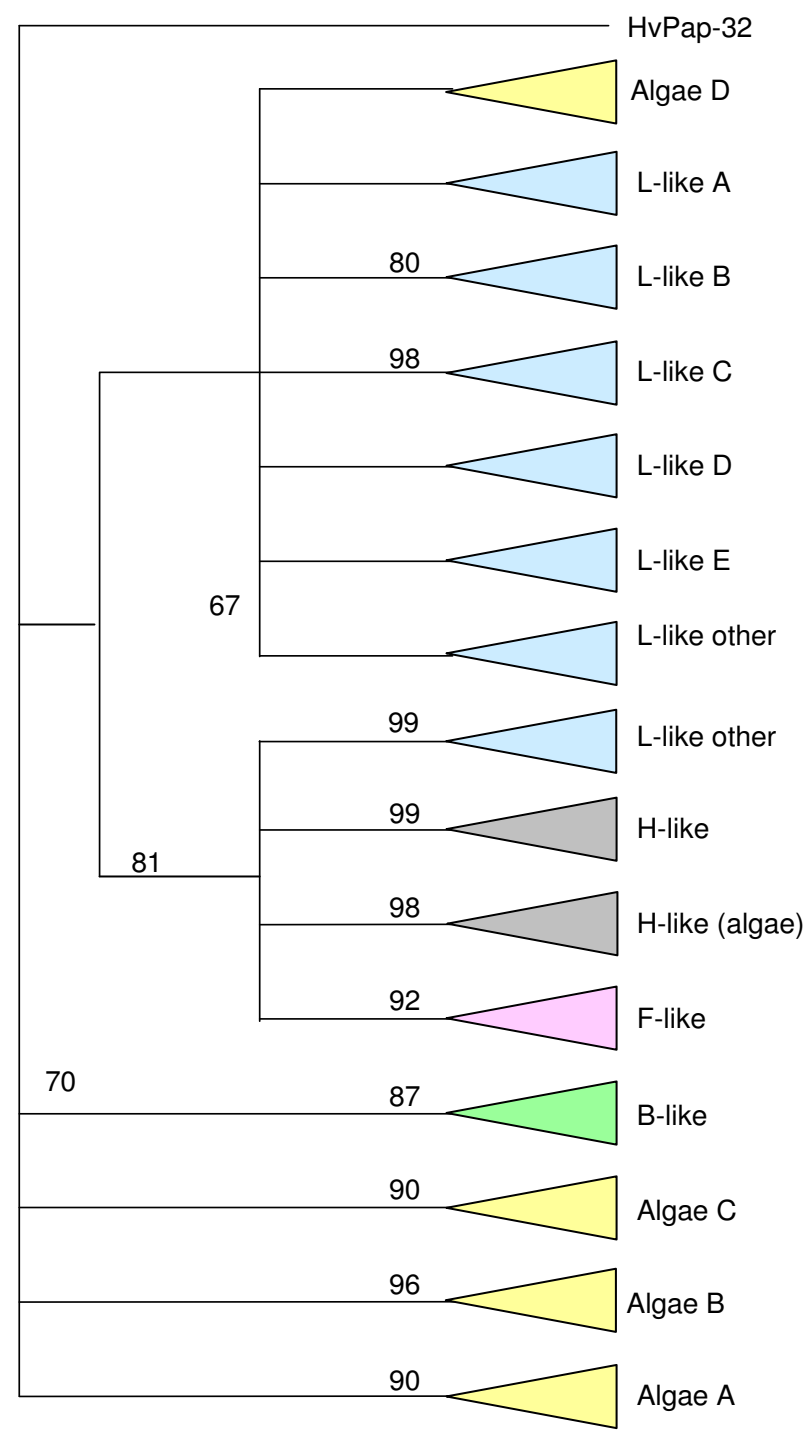

Figure 2

Schematic representation of the cladogram of cysteine proteinases CI A from algae to angiosperms. The amino acid sequences were aligned by MUSCLE and analysed with the PhyML method. Approximate likelihood-ratio test values $>\mathbf{8 0} \%$ are indicated. F-like, cathepsin F-like (pink); $\mathrm{H}$-like, cathepsin $\mathrm{H}$-like (grey); B-like, cathepsin B-like (green); L-like, cathepsin L-like (blue). Other cysteine proteinases CIA from algae (yellow). Vc, Volvox carteri.

ratio test values (aLRT) higher than $80 \%$, mainly among putatively orthologous monocot or dicot proteins. On the contrary, no orthology could be detected among moss and spikemoss sequences and the rest of $\mathrm{C} 13$ proteinases.

\section{Evolution of cystatins in plants}

Once known the evolution of C1A and C13 cysteine proteinases along the different plant clades, we seek to know

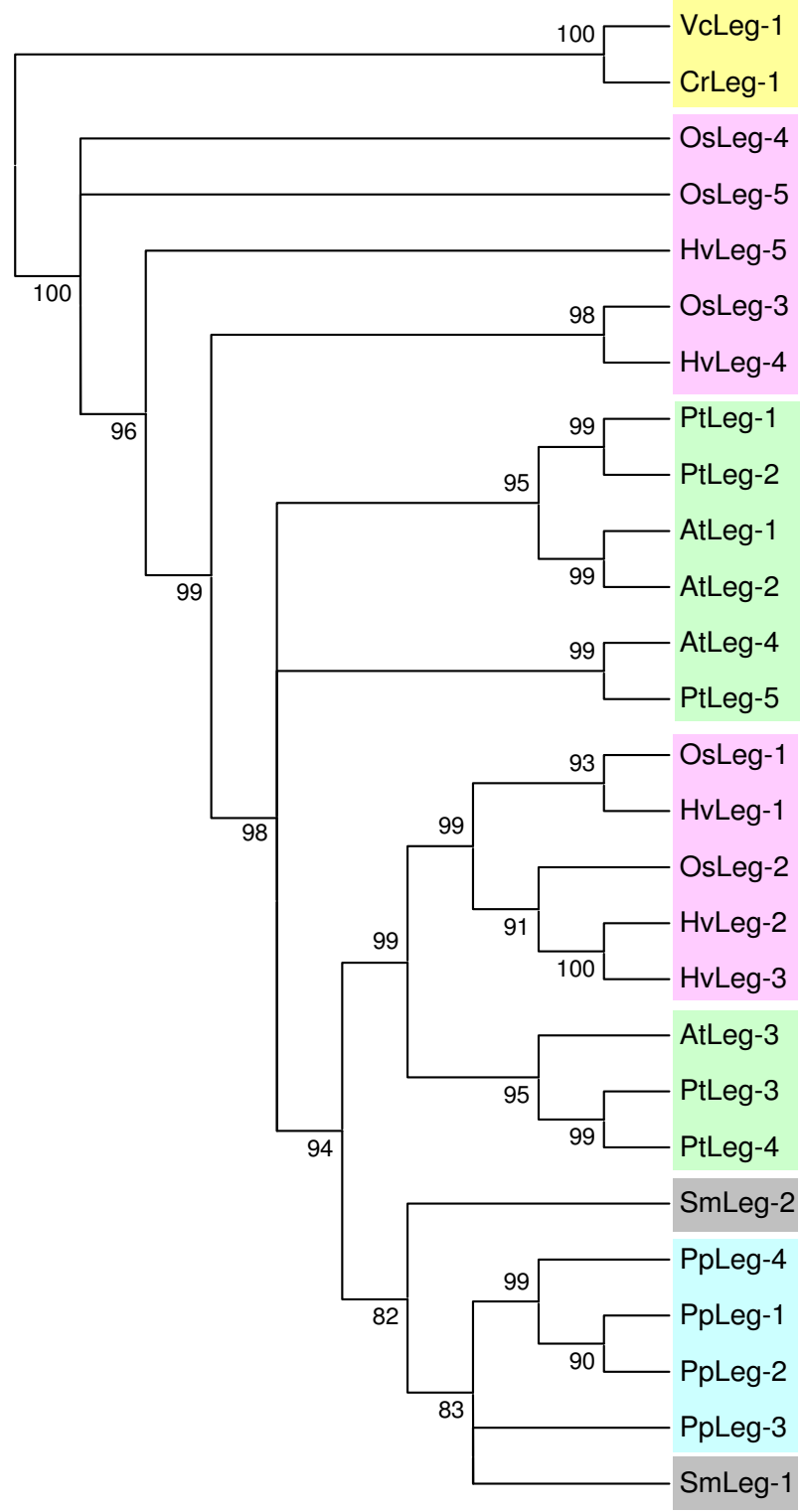

Figure 3

Cladogram of the cysteine proteinases $\mathrm{Cl} 3$ from algae to angiosperms. The amino acid sequences were aligned by MUSCLE and analysed with the PhyML method. Approximate likelihood-ratio test values $>80 \%$ are indicated. Green, dicot proteins (At, Arabidopsis thaliana; Pt, Populus tricocarpa); pink, monocot proteins (Os, Oryza sativa; Hv, Hordeum vulgare); blue, moss proteins ( $\mathrm{Pp}$, Physcomitrella patens); grey, spikemoss proteins (Sm, Selaginella moellendorffii); yellow, algal proteins $(\mathrm{Cr}$, Chlamidomonas reinhardtii; Vc, Volvox carteri).

how their inhibitory proteins, the cystatins, could have evolved. Likewise, the cystatin amino acid sequences and the sequences of the carboxy terminal extensions were aligned by MUSCLE (see Additional file 1), and phyloge- 
nies were constructed by the maximum likelihood PhyML method. When the main cystatin body was compared, omitting the signal peptides and the C-terminal extensions (Fig. 4), the cystatins from Chlorophyceae were the most different to the other cystatin sequences. The rest of sequences, with the exception of PtCPI-6, AtCYS5 and AtCYS4, could be distributed in three groups, one of them containing the five cystatins from the moss, the two cystatins from the spikemoss and several cystatins from all the angiosperm species. A second group was formed by mono or dicotyledoneous sequences, and only barley and rice sequences were located in the third group. When the Cterminal extensions were compared, the cystatins from the moss and the spikemoss were grouped separately from the angiosperm sequences (Fig. 5). The low number of 3' extended cystatins in completely sequenced genomes led us to compare the sequences obtained for other plant species from the TIGR Plant Transcript Assemblies (Fig. 6). The PhyML obtained tree resembles the evolutionary relationships among plant species, from algae to angiosperms, which denotes the ancestral characteristic of these C-terminal extensions. Interestingly, when the cDNA sequences of the cystatins with a C-terminal extension of the two algae species (one Chlorophyceae and one Trebuxiophyceae) were revised, the two main domains of the protein, the cystatin and the legumain inhibitory domain were separated by a stop codon, which was not present in the rest of carboxy extended proteins from plants.

\section{Discussion}

Nowadays, comparative genomic analysis has become as a powerful tool to discover evolutionary relationships between protein families. In this work, we analysed the evolutionary relationships among cystatins and their putative target enzymes, the cysteine proteinases $\mathrm{C} 1 \mathrm{~A}$ and C13 in different fully sequenced clades that appeared during plant evolution. To date, a comprehensive analysis of cystatin families had been only done in the completed genomes of $A$. thaliana and O. sativa [43]. For C1A cysteine proteinases, only the protein families in Arabidopsis and $P$. trichocarpa had been previously described $[44,45]$. At present, the complete protein family of C13 proteinases had not been analysed in any plant species.

The present phylogenetic study of phytocystatins indicates that this subfamily might have evolved from a common ancestor. This ancestral protein could have been lost in some algal lineages as in the Prasinophytes, and maintained in some other as the Chlorophyceae. However, the algal cystatins differ from the rest of the PhyCys in the presence of two cysteine residues, and the absence of the consensus motif [LVI]- [AGT]- [RKE]- [FY]- [AS]- [VI]-x[EDQV]- [HYFQ]-N specific to PhyCys [3]. Later on, this protein family would have expanded in the different tax-

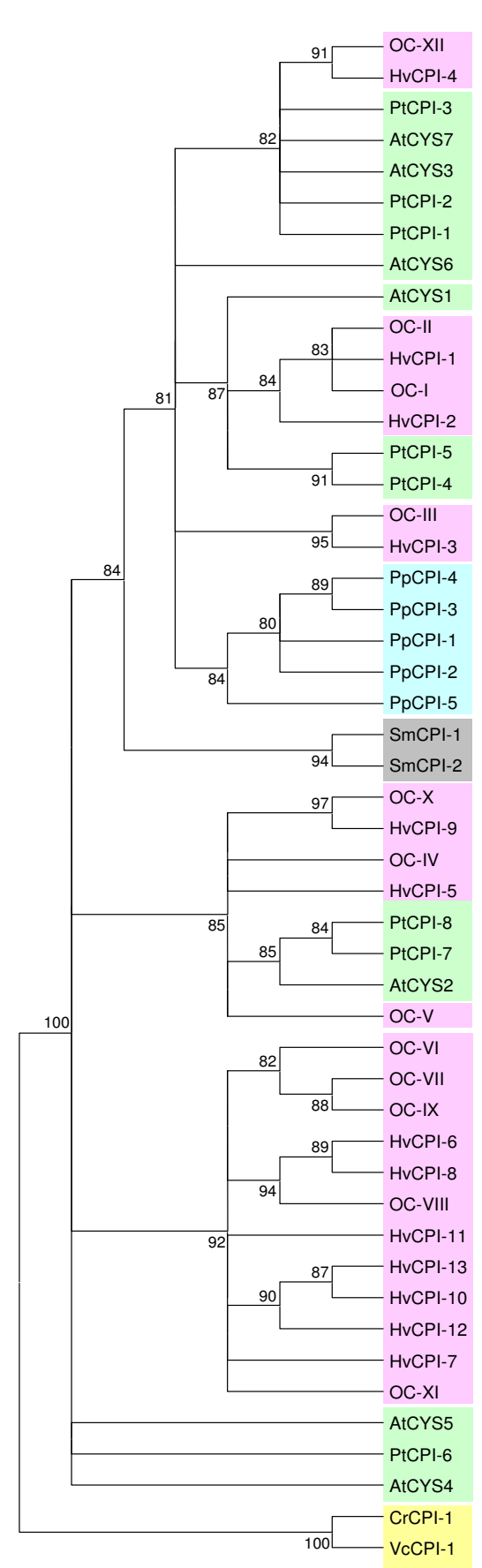

\section{Figure 4}

Cladogram of the cystatins from algae to

angiosperms. The amino acid sequences were aligned by MUSCLE and analysed with the PhyML method. Approximate likelihood-ratio test values $>80 \%$ are indicated. Green, dicot proteins (At, Arabidopsis thaliana; Pt, Populus tricocarpa); pink, monocot proteins (Os, Oryza sativa; Hv, Hordeum vulgare); blue, moss proteins ( $\mathrm{Pp}$, Physcomitrella patens); grey, spikemoss proteins ( $\mathrm{Sm}$, Selaginella moellendorffii); yellow, algal proteins ( $\mathrm{Cr}$, Chlamidomonas reinhardtii; $\mathrm{Vc}$, Volvox carteri). 


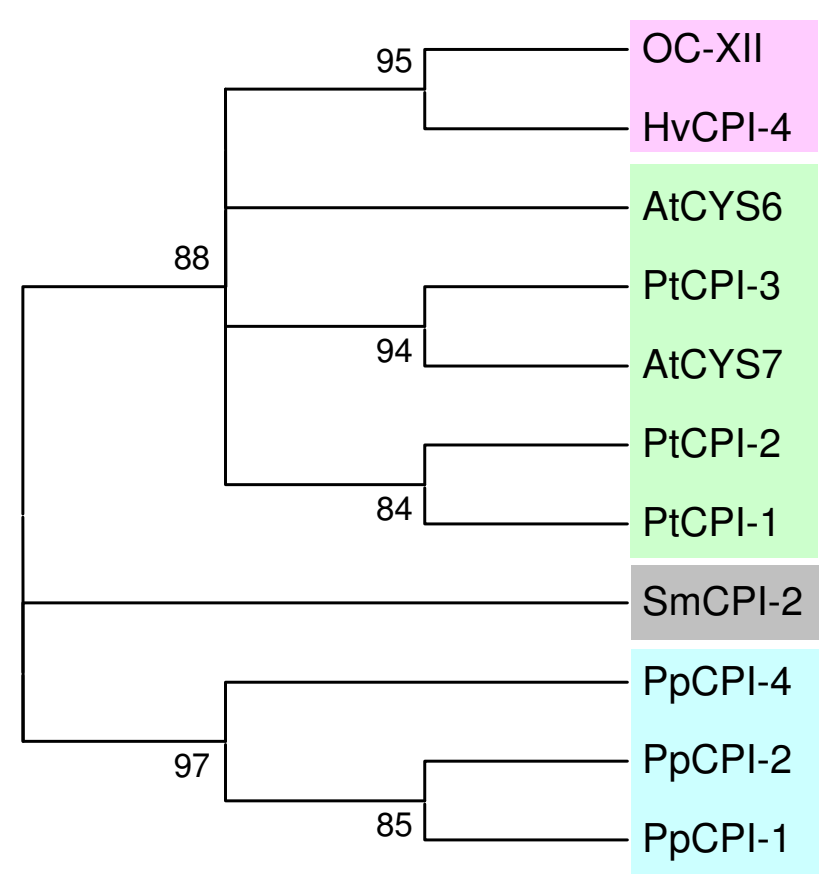

Figure 5

Cladogram of the carboxy terminal domain of long cystatins from the moss to angiosperms. The amino acid sequences were aligned by MUSCLE and analysed with the PhyML method. Approximate likelihood-ratio test values $>80 \%$ are indicated. Green, dicot proteins (At, Arabidopsis thaliana; Pt, Populus tricocarpa); pink, monocot proteins (Os, Oryza sativa; $\mathrm{Hv}$, Hordeum vulgare); blue, moss proteins (Pp, Physcomitrella patens); grey, spikemoss proteins (Sm, Selaginella moellendorffii).

onomic groups of vascular plants by recurrent duplication events in the course of evolution. Whether the ancestral cystatin was able to inhibit both C1A and C13 cysteine proteinases remains unknown. The C. reinhardtii and $V$. carteri cystatins did not posses the carboxy terminal extension present in the phytocystatins that inhibit legumainlike cysteine proteinases [6]. However, some animal cystatins are able to inhibit this kind of proteinases using the main core of the cystatin domain [46]. Since both algae possess one legumain-like protein, this second possibility cannot be discarded. Interestingly, two cystatins expressed by the algae Scenedesmus obliquus and Heliosporidium sp ex Simulium jonesii, belonging to the related algae families Chlorophyceae and Trebouxiophyceae did have the carboxy terminal extension with the legumain inhibitory motif. This carboxy terminal extensions are recognized by the Pfam motif database [47] as a cystatin domain, which suggest that C-extensions of plant cystatins are degenerated parts of a dimeric cystatin that probably originated by gene duplication followed by divergent evolution. Curiously, a stop codon is located between the two domains of these algal proteins. Since they are correctly expressed, the whole protein could be translated by readthrough the stop codon. In eukaryotes, readthrough is mainly due to decoding of the internal stop as normal amino acid or a noncanonical one, as seen for $C$. reinhardtii [48], suppression by a natural non-sense suppressor tRNA $[49,50]$, or a stable hairpin loop structure downstream of the stop codon [51].

With the exception of $C$. reinhardtii and $V$. carteri, the presence of cystatins with the C-terminal extension and legumain-like proteinases is well correlated. The Prasinophytes O. tauri and O. lucimarinus are devoid of both whereas vascular plants posses several members of these two protein families. Legumain-like cysteine proteinases are a small family in plants since no more than five members of this subfamily are present in any species. Likewise, the number of cystatins with the C-terminal extension is small, ranging from one to three. In higher plants, legumains have been involved in important physiological processes. They are implicated in the activation of papain-like cysteine proteinases to degrade storage proteins in the seed $[39,40,52]$, and in defense against pathogens executing programmed cell death [41,42]. However, the restricted activity of these proteinases, which have not been related to an elevated number of physiological processes, could account for the small number of members of this protein family.

The case of the papain-like cysteine proteinases was significantly different. These proteins have been involved in many physiological processes in vascular plants, including protein processing in the seed, senescence and programmed cell death, pollen development, and defence against pathogens $[11,27,28,31,33,53]$. In algae, the large number of C1A proteinases, ranging from 9 to 14 , suggests an important role of these proteins in their life cycle. However, there is only one report concerning the physiological roles of C1A cysteine proteinases. A protein, VcPap-11, is induced by the sex-inducing pheromone of the multicellular green algae $V$. carteri [54]. Interestingly, whereas the C1A proteinases from vascular plants are all related to mammalian cathepsins from the groups $\mathrm{B}, \mathrm{F}, \mathrm{H}$ and $\mathrm{L}$, a large number of algal C1A members could not be assigned to these groups, including VcPap- 11 . This suggests specialized functions in algae for these proteins not present in vascular plants, such as the response to sexinducing pheromones. In angiosperms, the number of putative C1A cysteine proteinases dramatically increased (mainly L-like) which could also mean a specialization of these proteinases to the new functions required in these plants. In fact, Arabidopsis AtPap-14 (XCP1) and AtPap15 (XCP2), which belong to the angiosperm subgroup $\mathrm{B}$ of the L-like cathepsins, are expressed in the tracheary elements of the xylem [55]. Similarly, proteins of the L-like 


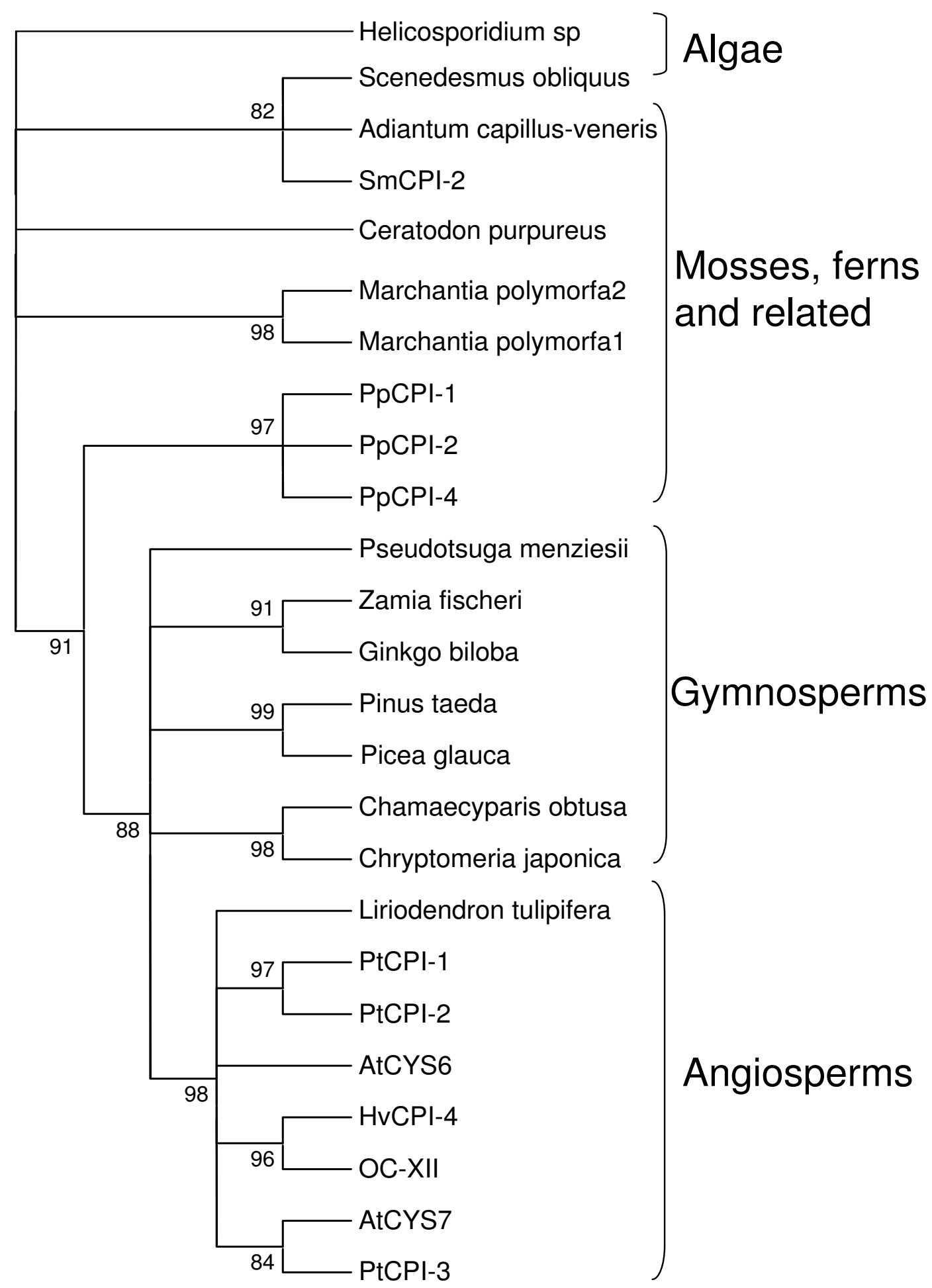

Figure 6

Cladogram of the carboxy terminal domain of long cystatins deduced from transcript assemblies of different Viridiplantae clades. The amino acid sequences were aligned by MUSCLE and analysed with the PhyML method. Approximate likelihood-ratio test values $>80 \%$ are indicated. At, Arabidopsis thaliana; Pt, Populus tricocarpa; Os, Oryza sativa; Hv, Hordeum vulgare; Pp, Physcomitrella patens; Sm, Selaginella moellendorffii. 
subgroup D formed by $\mathrm{C} 1 \mathrm{~A}$ sequences from algae to angiosperms could have evolved to achieve specific functions of higher plants. OsPap-24 and OsPap-18 (Oryzains $\mathrm{a}$ and $\mathrm{b}$ ) have been involved in seed storage mobilization, and OsPap-19 (OsCPI1) in pollen development $[53,56]$. Unfortunately, the function of the majority of these proteinases remains enigmatic, and testing for subfunctionalization and neofunctionalization events following gene duplication exceeds the scope of this project.

\section{Conclusion}

Comparative genomic analyses have provided us valuable insights into the conservation and evolution of the cystatin inhibitory family and their putative targets, the cysteine proteinases from families C1A and C13. A phylogenetic analysis of these gene families in representative species of different plant taxonomic groups has permitted us to state that whereas papain-like cysteine proteinases are present in all groups, cystatins and legumain-like proteins are absent in some basal groups. Moreover, in all these families, gene duplication events are associated with the increasing structural and functional complexities acquired in land plants. Thus, the complex functional relationship between both families of proteins must be the result of a coevolutionary process that might have occurred during the evolution of basal and land plants.

\section{Methods}

\section{Genome databases searches}

Blast searches for C1A and C13 cysteine proteinases and for cystatins were performed in publicly available genome databases. Sequences for Oryza sativa ssp. japonica (rice annotation release 5) were obtained at The Institute for Genomic Research (TIGR) [57]. Sequences for Arabidopsis thaliana were identified by searching The Arabidopsis Information Resource (TAIR) database (TAIR7 genome release) [58]. Searches for algae, moss, spikemoss and poplar sequences were carried out at the DOE Joint Genome Institute (JGI) [59], using the current releases: Chlamydomonas reinhardtii v3.0 [60]; Volvox carteri f. nagariensis v1.0 [61]; Ostreococcus lucimarinus v2.0 [62]; Ostreococcus tauri v2.0 [63]; Physcomitrella patens ssp. patens v1.1 [64]; Sellaginella moellendorffii v1.0 [65]; Populus trichocarpa v1.1 [66]. Information about gene models for all these proteins is compiled in Tables I, III and IV from Additional file 3.

\section{Screening of barley EST and cDNA libraries}

C1A, C13 cysteine proteinases and cystatin sequences were used to search for ESTs or cDNA sequences in publicly available libraries of barley using the tblastn program at the DNA Data Bank of Japan [67] and the Okayama University Barley Database [68]. Selected ESTs were obtained and sequenced in both strands using vector-specific primers using an automated DNA sequencer (ABI
PRISM TM 3100; Perkin Elmer-Applied Biosystems). Accession numbers are in Tables I, III and IV from additional file 3 .

\section{Searches for expressed genes in plants}

C1A, C13 cysteine proteinases and cystatin sequences were used to search the TIGR plant transcript assemblies (TA) database [69]. The sequences that are used to build the plant TAs are expressed transcripts collected from dbEST (ESTs) and the NCBI GenBank nucleotide database. Sequences are initially clustered based on an allagainst-all comparisons using Megablast. The initial clusters are assembled to generate consensus sequences using CAP3. Assembly criteria include a 50 bp minimum match, $95 \%$ minimum identity in the overlap region and $20 \mathrm{bp}$ maximum unmatched overhangs. Any EST/cDNA sequences that are not assembled into TAs are included as singletons. TAs from 252 plant species from unicellular organisms to flowering plants are included in this database. Transcript assembly accessions for cystatin 3' extended genes are shown in Table II from Additional file 3.

\section{Phylogeny of plants}

The phylogenetic tree used to the survey of the evolution of C1A, C13 cysteine proteinases and cystatins was based on the Viridiplantae tree of the Tree of Life Web Project (ToL) [70], which is a collaborative effort of biologists from around the world to provide information about the diversity of organisms on Earth, their evolutionary history (phylogeny), and characteristics.

\section{Protein alignments and Phylogenetic trees}

Alignments of the amino acid sequences were performed using the default parameters of MUSCLE version 3.6 [71]. For cystatins and cysteine proteinases C13 controversial models were not detected and the whole amino acid sequences were aligned. For cysteine proteinases C1A, presumed wrong models were detected and protein alignments were restricted to the part of the protein that was not controversial in any model and conserves the characteristic features of papain-like cysteine proteinases (from 25 amino acids before the putative location of the cysteine reactive residue until 18 amino acids after the asparagine reactive residue). Alignments ambiguities and gaps were excluded from phylogenetic analysis using GBLOCKS version 0.91b [72]. Phylogenetic and molecular evolutionary analyses were conducted using the programs PhyML and MEGA version 4.0 [73-75]. The displayed C1A, C13 cysteine proteinases and cystatin trees were derived using a maximum likelihood PhyML method using the WAG substitution model and a BIONJ starting tree. The approximate likelihood-ratio test (aLRT) based on a ShimodairaHasegawa-like procedure was used as statistical test for non-parametric branch support [76]. Displayed cladog- 
rams are condensed trees with cut-off aLRT values of $80 \%$. All families were also analysed with the Maximum parsimony, Unweighted Pair Group Method with Arithmetic Mean (UPGMA), and the Neighbour-Joining algorithms, and with different gap penalties. No significant differences in the tree topologies were detected.

\section{Identification of conserved motifs}

The deduced protein sequences of the putative $\mathrm{H}$ and Llike cathepsins were analysed by means of the MEME program $[77,78]$. Default parameters were used, except that the minimal and maximal motif widths were set to 6 and 12 amino acids, respectively, and the maximum number of motifs to find was defined as 50 .

\section{Authors' contributions}

MM designed the study, carried out the sequence analysis and interpretation of the results and drafted the manuscript. ID participated in the design of the study and the interpretation of the results and critically revised the manuscript.

\section{Additional material}

\section{Additional File 1}

Alignments performed using the MUSCLE program of the amino acid sequences corresponding to the proteins used in this study.

Click here for file

[http://www.biomedcentral.com/content/supplementary/14712148-8-198-S1.doc]

\section{Additional File 2}

Complete cladogram of cysteine proteinases C1A from algae to angiosperms from which Figure 1 is derived. The amino acid sequences were aligned by MUSCLE and analysed with the PhyML method. Approximate likelihood-ratio test values $>80 \%$ are indicated.

Click here for file

[http://www.biomedcentral.com/content/supplementary/14712148-8-198-S2.pdf]

\section{Additional File 3}

Information about gene models and accession numbers corresponding to the proteins used in this study.

Click here for file

[http://www.biomedcentral.com/content/supplementary/14712148-8-198-S3.doc]

\section{Acknowledgements}

The financial support from the Ministerio de Educación y Ciencia (BFU2005-00603) is gratefully acknowledged.

\section{References}

I. MEROPS: the peptidase database [http://merops.sanger.ac.uk]

2. Rawlings ND, Morton FR, Kok CY, Kong J, Barrett AJ: MEROPS: the peptidase database. Nucleic Acids Res 2008, 36:D320-D325.
3. Margis R, Reis EM, Villeret V: Structural and phylogenetic relationships among plant and animal cystatins. Arch Biochem Biophys 1998, 359:24-30.

4. Stubbs MT, Laber B, Bode W, Huber R, Jerala R, Lenarcic B, Turk V: The refined $2.4 \mathrm{~A} X$-ray crystal structure of recombinant human stefin $B$ in complex with the cysteine proteinase papain: a novel type of proteinase inhibitor interaction. $E M B O$ J 1990, 9:1939-1947.

5. Jenko S, Dolenc I, Guncar G, Dobersek A, Podobnik M, Turk D: Crystal structure of Stefin $A$ in complex with cathepsin $\mathbf{H}$ : $\mathbf{N}$ terminal residues of inhibitors can adapt to the active sites of endo- and exo-peptidases. J Mol Biol 2003, 326:875-885.

6. Martinez M, Diaz-Mendoza M, Carrillo L, Diaz I: Carboxy terminal extended phytocystatins are bifunctional inhibitors of papain and legumain cysteine proteinases. FEBS Let 2007, 58 I:29|4-29|8.

7. Arai S, Matsumoto I, Emori Y, Abe K: Plant seed cystatins and their target enzymes of endogenous and exogenous origin. J Agric Food Chem 2002, 50:6612-66I7.

8. Corre-Menguy F, Cejudo FJ, Mazubert C, Vidal J, Lelandais-Biere C Torres G, Rode A, Hartmann C: Characterization of the expression of a wheat cystatin gene during caryopsis development. Plant Mol Biol 2002, 50:687-698.

9. Martinez M, Rubio-Somoza I, Carbonero P, Diaz I: A cathepsin Blike cysteine proteinase gene from Hordeum vulgare (gene Cat $B$ ) induced by $\mathbf{G A}$ in aleurone cells is under circadian control in leaves. J Exp Bot 2003, 54:95I-959.

10. Hong KH, Hwang JE, Lim C], Yang KA, Jin ZL, Kim CY, Koo JC, Cheng WS, Lee KO, Lee SY, Cho MJ, Lim CO: Over-expression of Chinese cabbage phytocystatins I retards seed germination in Arabidopsis. Plant Science 2007, I 72:556-563.

II. Solomon M, Belenghi B, Delledonne M, Levine A: The involvement of cysteine proteinases and proteinase inhibitor genes in programmed cell death in plants. Plant Cell I999, I I:43 |-444.

12. Belenghi B, Acconcia F, Trovato M, Perazzolli M, Bocedi A, Polticelli F, Ascenzi P, Delledonne M: AtCYSI, a cystatin from Arabidopsis thaliana, suppresses hypersensitive cell death. Eur I Biochem 2003, 270:2593-2604.

13. Koiwa H, Shade RE, Zhu-Salzman K, D'Urzo MP, Murdock LL, Bressan RA, Hasegawa PM: A plant defensive cystatin (soyacystatin) targets cathepsin L-like digestive cysteine proteinases (DvCALs) in the larval midgut of western corn rootworm (Diabrotica virgifera virgifera). FEBS Let 2000, 47 I:67-70.

14. Outchkourov NS, de Kogel WJ, Wiegers GL, Abrahamson M, Jongsma MA: Engineered multidomain cysteine protease inhibitors yield resistance against western flower thrips (Frankliniella occidentalis) in greenhouse trials. Plant Biotechnol J 2004, 2:449-458.

15. Alvarez-Alfageme F, Martinez M, Pascual-Ruiz S, Castañera P, Diaz I, Ortego F: Effects of potato plants expressing a barley cystatin on the predatory bug Podisus maculiventris via herbivorous prey feeding on the plant. Transgenic Res 2007, I 6: I- I3.

16. Urwin PE, Atkinson HJ, Waller DA, McPherson MJ: Engineered oryzacystatin-I expressed in transgenic hairy roots confers resistance to Globodera pallida. Plant J I995, 8: | 2 | - I $3 \mid$.

17. Gutierrez-Campos R, Torres-Acosta J, Saucedo-Arias LJ, Gomez-Lim MA: The use of cysteine proteinase inhibitors to engineer resistance against potyviruses in transgenic tobacco plants. Nat Biotechnol 1999, 17:1223-1226.

18. Ribeiro APO, Pereira EJG, Galvan TL, Picando MC, Picoli EAT, da Silva $\mathrm{DJH}$, Fári MG, Otoni WC: Effect of eggplant transformed with oryzacystatin gene on Myzus persicae and Macrosiphum euphorbiae. J Appl Entomol 2006, I30:84-90.

19. Pernas M, Lopez-Solanilla E, Sanchez-Monge R, Salcedo G, RodriguezPalenzuela P: Antifungal activity of a plant cystatin. Mol PlantMicrobe Interact 1999, I 2:624-627.

20. Martinez M, Lopez-Solanilla E, Rodriguez-Palenzuela $P$, Carbonero $P$ Diaz I: Inhibition of plant-pathogenic fungi by the barley cystatin HvCPI (gene lcy) is not associated with its cysteineproteinase inhibitory properties. Mol Plant-Microbe Interact 2003, I 6:876-883.

21. Martinez M, Abraham Z, Gambardella M, Echaide M, Carbonero P, Diaz I: The strawberry gene Cyfl encodes a phytocystatin with antifungal properties. J Exp Bot 2005, 56:|82|-|829.

22. Yang $\mathrm{AH}$, Yeh $\mathrm{KW}$ : Molecular cloning, recombinant gene expression, and antifungal activity of cystatin from taro 
(Colocasia esculenta cv. Kaosiung no.l). Planta 2005, 221:493-501.

23. Abraham Z, Martinez M, Carbonero P, Diaz I: Structural and functional diversity within the cystatin gene family of Hordeum vulgare. J Exp Bot 2006, 57:4245-4255

24. Kamphuis IG, Drenth J, Baker EN: Thiol proteinases. Comparative studies based on the high-resolution structures of papain and actinidin, and on amino acid sequence information for cathepsins B and $\mathbf{H}$, and stem bromelain. J Mol Biol 1985, 182:317-329.

25. Wiederanders B, Kaulmann G, Schilling K: Functions of propeptide parts in cysteine proteinases. Curr Protein Pept Sci 2003, 4:309-326.

26. Jung R, Scott MP, Nam YW, Beaman TW, Bassuner R, Saalbach I, Muntz K, Nielsen NC: The role of proteolysis in the processing and assembly of I IS seed globulins. Plant Cell 1998, 10:343-357.

27. Gruis D, Schulze J, Jung R: Storage protein accumulation in the absence of the vacuolar processing enzyme family of cysteine proteinases. Plant Cell 2004, 16:270-290.

28. Gruis DF, Selinger DA, Curran JM, Jung R: Redundant proteolytic mechanisms process seed storage proteins in the absence of seed-type members of the vacuolar processing enzyme family of cysteine proteinases. Plant Cell 2002, 14:2863-2882.

29. Kiyosaki T, Matsumoto I, Asakura T, Funaki J, Kuroda M, Misaka T, Arai S, Abe K: Gliadain, a gibberellin-inducible cysteine proteinase occurring in germinating seeds of wheat, Triticum aestivum $L$., specifically digests gliadin and is regulated by intrinsic cystatins. FEBS J 2007, 274:1908-1917.

30. Ueda T, Seo S, Ohashi Y, Hashimoto J: Circadian and senescenceenhanced expression of a tobacco cysteine proteinase gene. Plant Mol Biol 2000, 44:649-657.

31. Xu ZF, Chye ML, Li HY, Xu FX, Yao KM: G-box binding coincides with increased Solanum melogena cysteine proteinase expression in senescent fruits and circadian-regulated leaves. Plant Mol Biol 2003, $51: 9-19$.

32. Khanna-Chopra R, Srivalli B, Ahlawat YS: Drought induces many forms of cysteine proteinases not observed during natural senescence. Biochem Biophys Res Commun 1999, 255:324-327.

33. Hoorn $R$ van der, Jones J: The plant proteolytic machinery and its role in defence. Curr Opin Plant Biol 2004, 7: I-8.

34. Gilroy EM, Hein I, Hoorn R van der, Boevink PC, Venter E, McLellan H, Kaffarnik F, Hrubikova K, Shaw J, Holeva M, López EC, BorrasHidalgo O, Pritchard L, Loake G], Lacomme C, Birch PR: Involvement of cathepsin $B$ in the plant disease resistance hypersensitive response. Plant J 2007, 52: I- I3.

35. Chen JM, Rawlings ND, Stevens RA, Barrett AJ: Identification of the active site of legumain links it to caspases, clostripain and gingipains in a new clan of cysteine endopeptidases. FEBS Lett 1998, 441:361-365.

36. Chen JM, Fortunato M, Barrett AJ: Activation of human prolegumain by cleavage at a $\mathrm{C}$-terminal asparagine residue. Biochem J 2000, 352:327-334.

37. Hara-Nishimura I, Inoue K, Nishimura M: A unique vacuolar processing enzyme responsible for conversion of several proprotein precursors into the mature forms. FEBS Lett 1991, 294:89-93.

38. Hara-Nishimura I, Shimada T, Hiraiwa N, Nishimura M: Vacuolar processing enzymes responsible for maturation of seed proteins. J Plant Physiol 1995, 145:632-640.

39. Okamoto T, Minamikawa T: Molecular cloning and characterization of Vigna mungo processing enzyme I (VmPE-I), an asparaginyl endopeptidase possibly involved in post-translational processing of a vacuolar cysteine endopeptidase (SHEP). Plant Mol Biol 1999, 39:63-73.

40. Kato $\mathrm{H}$, Sutoh $\mathrm{K}$, Minamikawa T: Identification, cDNA cloning and possible roles of seed-specific rice asparaginyl endopeptidase, REP-2. Planta 2003, 21 7:676-685.

4I. Hatsugai N, Kuroyanagi M, Yamada K, Meshi T, Tsuda S, Kondo M, Nishimura M, Hara-Nishimura I: A plant vacuolar proteinase, VPE, mediates virus-induced hypersensitive cell death. Science 2004, 305:855-858.

42. Rojo E, Martin R, Carter C, Zouhar J, Pan S, Plotnikova J, Jin H, Paneque M, Sanchez-Serrano J], Baker B, Ausubel FM, Raikhel NV: VPEgamma exhibits a caspase-like activity that contributes to defense against pathogens. Curr Biol 2004, 14:1897-1906.
43. Martinez M, Abraham Z, Carbonero P, Diaz I: Comparative phylogenetic analysis of cystatin gene families from arabidopsis, rice and barley. Mol Genet Genomics 2005, 273:423-432.

44. Beers EP, Jones AM, Dickerman AW: The S8 serine, CI A cysteine and AI aspartic proteinase families in Arabidopsis. Phytochemistry 2004, 65:43-58.

45. García-Lorenzo M, Sjödin A, Jansson S, Funk C: Proteinase gene families in Populus and Arabidopsis. BMC Plant Biol 2006, 6:30.

46. Alvarez-Fernandez M, Barrett AJ, Gerhartz B, Dando PM, Ni J, Abrahamson M: Inhibition of mammalian legumain by some cystatins is due to a novel second reactive site. J Biol Chem 1999, 274: 19195-19203.

47. Pfam [http://pfam.sanger.ac.uk]

48. Novoselov SV, Rao M, Onoshko NV, Zhi H, Kryukov GV, Xiang Y, Weeks DP, Hatfield DL, Gladyshev VN: Selenoproteins and selenocysteine insertion system in the model plant cell system, Chlamydomonas reinhardtii. EMBO J 2002, $21: 368 \mid-3693$.

49. Beier $\mathrm{H}$, Grimm $\mathrm{M}$ : Misreading of termination codons in eukaryotes by natural nonsense suppressor tRNAs. Nucleic Acids Res 200I, 29:4767-4782.

50. Liu Q, Xue Q: Computational identification and sequence analysis of stop codon readthrough genes in Oryza sativa. Biosystems 2004, 77:33-9.

5I. Steneberg P, Samakovlis C: A novel stop codon readthrough mechanism produces functional Headcase protein in Drosophila trachea. EMBO Rep 200I, 2:593-597.

52. Zakharov A, Carchilan M, Stepurina T, Rotari V, Wilson K, Vaintraub I: A comparative study of the role of the major proteinases of germinated common bean (Phaseolus vulgaris L.) and soybean (Glycine max (L.) Merrill) seeds in the degradation of their storage proteins. J Exp Bot 2004, 55:224I-2249.

53. Lee $S$, Jung $K H$, An G, Chung YY: Isolation and characterization of a rice cysteine proteinase gene, OsCPI, using T-DNA gene-trap system. Plant Mol Biol 2004, 54:755-765.

54. Amon $P$, Haas $E$, Sumper $M$ : The sex-inducing pheromone and wounding trigger the same set of genes in the multicellular green alga Volvox. Plant Cell 1998, 10:78I-789.

55. Funk V, Kositsup B, Zhao C, Beers EP: The Arabidopsis xylem peptidase XCPI is a tracheary element vacuolar protein that may be a papain ortholog. Plant Physiol 2002, I 28:84-94.

56. Watanabe H, Abe K, Emori Y, Hosoyama H, Arai S: Molecular cloning and gibberellin-induced expression of multiple cysteine proteinases of rice seeds (oryzains). J Biol Chem I99I, 266: 16897-16902.

57. The Institute for Genomic Research (TIGR) [http:// www.tigr.org]

58. The Arabidopsis Information Resource (TAIR) [http:// www.arabidopsis.org]

59. The DOE Joint Genome Institute (JGI) [http://
] www.jgi.doe.gov]

60. Chlamydomonas reinhardtii v3.0 [http://genome.jgi-psf.org/ Chlre3]

6I. Volvox carteri f. nagariensis vl.0 [http://genome.jgi-psf.org/ Volcal]

62. Ostreococcus lucimarinus v2.0 [http://genome.jgi-psf.org/ Ost990I 3]

63. Ostreococcus tauri v2.0 [http://genome.jgi-psf.org/Ostta4]

64. Physcomitrella patens ssp. Patens vi.l [http://genome.jgipsf.org/Phypal I]

65. Sellaginella moellendorffii v1.0 [http://genome.jgi-psf.org/Selmol]

66. Populus trichocarpa vl.I [http://genome.jgi-psf.org/Poptrl I]

67. The DNA Data Bank of Japan [http://www.ddbj.nig.ac.jp/Wel come-e.html]

68. The Okayama University Barley Database [http://www.shi gen.nig.ac.jp/barley]

69. The TIGR plant transcript assemblies (TA) database [http:l /plantta.tigr.org]

70. The Tree of Life Web Project (ToL) [http://www.tolweb.org/ tree]

71. Edgar RC: MUSCLE: multiple sequence alignment with high accuracy and high throughput. Nucleic Acids Res 2004, 32:1792-1797.

72. Castresana J: Selection of conserved blocks from multiple alignments for their use in phylogenetic analysis. Mol Biol Evol $2000,17: 540-552$. 
73. Guindon S, Gascuel O: A simple, fast, and accurate algorithm to estimate large phylogenies by maximum likelihood. Syst Biol 2003, 52:696-704.

74. MEGA 4: Molecular Evolutionary Genetics Analysis [http:// www.megasoftware.net]

75. Tamura K, Dudley J, Nei M, Kumar S: MEGA4: Molecular Evolutionary Genetics Analysis (MEGA) software version 4.0. Mol Biol Evol 2007, 24:1596-1599.

76. Anisimova A, Gascuel O: Approximate likelihood ratio test for branchs: A fast, accurate and powerful alternative. Syst Biol 2006, 55:539-552.

77. Multiple Em for Motif Elicitation (MEME) [http:// meme.sdsc.edu/meme/meme.html]

78. Bailey TL, Elkan C: Fitting a mixture model by expectation maximization to discover motifs in biopolymers. Proc Int Conf Intell Syst Mol Biol 1994, 2:28-36.

Publish with Biomed Central and every scientist can read your work free of charge

"BioMed Central will be the most significant development for disseminating the results of biomedical research in our lifetime. "

Sir Paul Nurse, Cancer Research UK

Your research papers will be:

- available free of charge to the entire biomedical community

- peer reviewed and published immediately upon acceptance

- cited in PubMed and archived on PubMed Central

- yours - you keep the copyright

Submit your manuscript here:

http://www.biomedcentral.com/info/publishing_adv.asp
BiolMedcentral 